\title{
Modern Trends: The Impact of Social, Technological, and Economic Forces on Psychiatric Education and Training
}

\author{
Christopher R. Thomas ${ }^{1}$ Anthony L. Rostain ${ }^{2} \cdot$ Eugene V. Beresin ${ }^{3}$
}

Received: 12 September 2016 / Accepted: 19 September 2016/Published online: 19 October 2016

(C) Academic Psychiatry 2016

Education has evolved in the medical professions on the basis of the perceived needs for training, the availability of educational resources, and the methods judged most appropriate for teaching the necessary competencies for current clinical practice, research, and administration. According to current trends, a number of social, economic, and political forces are expected to have a major influence on undergraduate medical education (UME) and graduate medical education (GME) in the future. In recent years, most attention in medical education has focused on the rapid changes in the content, pedagogic models, and methods of evaluating students and residents. Significant changes in the organization, regulation, and support for medical education have also occurred, however, along with increased societal pressures for additional and new services. The impact of these factors on the current systems of care, educational platforms, and workforce are overlapping and complex. For the purpose of review and discussion in this editorial, we have grouped them into the categories of regulatory, financial, clinical learning environment, social, and information technology factors.

\section{Regulatory Factors}

Regulatory pressures at both state and national levels have long influenced UME and GME. The Association of

\section{Christopher R. Thomas}

crthomas@utmb.edu

\footnotetext{
University of Texas Medical Branch, Galveston, TX, USA

University of Pennsylvania Health System, Philadelphia, PA, USA

Harvard Medical School, Boston, MA, USA
}

American Medical Colleges (AAMC) was formed in 1876 primarily to reform and improve medical education. The American Medical Association (AMA) joined in this effort with the creation of the Council on Medical Education in 1904 [1]. Shortly after, in 1910, the Carnegie Foundation published the profoundly influential Flexner Report. This report led in part to the AMA establishing a program in 1914 to evaluate and approve residencies and fellowships. This responsibility for oversight and accreditation of GME was shifted to the specialty boards following their creation. The American Board of Psychiatry and Neurology (ABPN) was founded in 1934 to establish training standards and to credential practitioners [2]. The introduction of Medicare and federal funding for GME highlighted the need for the multiple accrediting councils and varying training standards to be revised to create a more uniform and organized approach. The Liaison Committee for Graduate Medical Education was established in 1972 and succeeded by the Accreditation Council for Graduate Medical Education (ACGME) in 1981. The US Department of Education in the 1980s required the use of outcome measures for any education program that received federal funding. This requirement was part of the impetus for the ACGME to create the Core Competencies and subsequently the Milestones for GME [3].

These regulatory agencies will continue to have a strong influence on medical education in the future, through accrediting training and setting requirements for graduation and postgraduate certification. The concern for the quality of medical education mirrors federal and state investment in ensuring practitioner competency in the interest of public health and safety. Such interest can be seen not only in the implementation of outcomes measures for training (ACGME Milestones) and certification (American Board of Medical Specialties [ABMS] through Maintenance of Certification [MOC]) [4] but also in the response to public concerns about 
physician error (e.g., the Institute of Medicine's duty hour requirements) [5]. The ACGME Program Requirements initially expected programs and institutions to train residents in fatigue awareness and to devise procedures to handle residents who were too tired to function safely. The Program Requirements became more specific on these concerns with the institution of duty hours, limiting the total number of hours not only for training but also for moonlighting that a program must track. These changes led to increases in patient care handoffs and subsequent ACGME Program Requirements on supervision and documentation of handoffs. Recent federal legislation, the Medicare Access and CHIPS Reauthorization Act of 2015 (MACRA), reflects a similar emphasis on the quality of care being provided by instituting payment-based quality measures, the Merit-Based Incentive Payment System (MIPS) [6]. Private insurance carriers will very likely follow this example. Medical education in the future will be shaped not only by concerns for the outcome of training in producing competent physicians but also by the preparation of those graduates to practice in health care systems measured by the quality of care provided. Toward that goal, the ACGME now requires residents and fellows to participate in institutional quality improvement projects during training so that graduates routinely evaluate practices that ensure patient safety and improve clinical outcomes. Although this requirement is sensible in the overall goals for improving medical education and patient care, it is another metric for programs to record and report and requires increased administrative and educational time for training. Most educators support the mission of ensuring quality of care and incorporating these requirements into training; the required education and reporting of outcomes have long been considered as unfunded mandates to training programs, however, because they have no means of subsidizing these increased efforts.

The implementation of the ACGME Next Accreditation System in 2013 signaled a major shift in GME oversight, moving from periodic review of individual programs every 3 to 5 years to a process of continuous oversight with annual reviews [7]. Although this shift provides a better means to detect problems or deficiencies quickly, it requires a constant flow of information from individual programs to the ACGME. Annual information uploads include not only updates of faculty and resident scholarly activity but also resident and faculty surveys. This shift, coupled with the introduction of competency-based outcomes in education, has resulted in a greater burden on both training program administration and faculty supervisors. Institutional time allotted for faculty development and for performing necessary evaluations of trainee competencies must compete with the increased demands for clinical productivity in training programs. The Next Accreditation System may ensure that programs are more closely monitored, but it has resulted in significantly more administrative load for programs to sustain.
Another continuing trend and influence involves how the overlap between agencies providing oversight functions of different aspects of medical education change and interact. A good example of this interaction is the recent consolidation of GME accreditation of training with the agreement of the ACGME and the American Osteopathic Association for oversight of programs for doctors of osteopathy medicine. Another is the decision by the ACGME to not accredit combined programs (other than internal medicine-pediatrics) and to resume accreditation for those combined programs involving psychiatry by the ABPN. The Liaison Committee on Medical Education, ACGME, and ABMS all have been discussing and reviewing their individual efforts to implement competency-based outcomes for education, but this discussion has not resulted in a common set of approaches or assessments for UME, GME, and MOC. Medical training has become much more detailed at each stage, leading to separation of training methods and discontinuity for learners as they move from one stage to the next. This separation also places a greater burden on institutions, with the administrative load of separate training processes for faculty supervisors dealing with UME, GME, and their own MOC.

\section{Economic Factors}

A 2016 report by the AAMC [8] states that there is at present, and there will continue to be over the next decade, an increasingly severe national shortage of physicians, particularly in primary care and numerous subspecialties. This shortage is primarily the result of an increasing demand for physicians because of the numbers of physicians retiring, the expansion and aging of the US population, and the increased access to health care offered by the Affordable Care Act (ACA). Unfortunately, the number of GME slots has been capped since the Balanced Budget Act of 1997. At the same time, the recent growth in US medical schools (both MD and DO programs) is leading to the lack of residency positions.

Psychiatry has long experienced a profound shortage in general psychiatry and all of its subspecialties. In child psychiatry, for example, an estimated 8300 child psychiatrists care for approximately 15 million youth with severe mental illness. The continuing shortages in the mental health workforce result in increasing demands on clinical services and faculty. To complicate matters, decreased reimbursement for clinical services results in increased workloads for faculty and monstrous new pressures on academic departments to garner funds for protected teaching time and scholarship. While faculty in teaching hospitals are experiencing expanding clinical responsibilities, the demands for accountable educational performance and assessment of medical student and resident Milestones by the AAMC and ACGME puts tremendous strain on the readiness and ability of faculty to teach or even 
have protected time for teaching. A noted historian of medical education, Kenneth M. Ludmerer, M.D., has observed that the commercialization of health care delivery has pressured clinical faculty members to the point where their availability to teach and supervise students and trainees has been seriously undermined [9].

Mental health - particularly that of children and familieshas received increasing public attention and is now widely viewed as in crisis. Furthermore, there is growing recognition that the lifetime prevalence of psychiatric disorders is about $24 \%$, with almost half beginning in childhood and adolescence [10]. These data fly in the face of extreme shortages not only, as noted, in workforce but also in hospital beds for children and adolescents, wraparound services, school-based programs, and hospital and community services to care for children, transitional-age youth, and families. Moreover, the current insurance system has vast constraints on comprehensive care for children and families, despite the movement toward parity - a movement that has fallen far short of what comparable care of mental illness with medical illness really means. The same features of unmet mental health needs and lack of a trained workforce exist for other psychiatric subspecialties, especially geriatric and addiction psychiatry.

In response to these facts, Congress is debating the Helping Families in Mental Health Crisis Act (H.R. 2646) [11], which would address increased coverage and services for children and adolescents, improved infrastructure overseeing evidence-based mental health care, and increased training for pediatric subspecialties. Even with greater numbers of beds, outpatient services, and training for existing personnel, however, there are no provisions at this time for increasing the number of GME slots for child and adolescent psychiatrists. Thus, the "good news" in increased awareness of mental health as a public health crisis and need for more services is dramatically offset by the reality that there is no plan to produce more mental health practitioners or to provide increased funding for research addressing the causes, evaluation, or treatment of child and adolescent psychiatric disorders or of psychiatric disorders in general.

Beyond constraints on teaching time, the National Institutes of Health (NIH) has dramatically reduced funding to medical centers, cutting back research and affiliated training and curtailing preparation of the next generation of researchers. Additionally, many academic institutions have set restrictions on clinical research with funding from the pharmaceutical industry and others in the private sector.

These two factors, decreased time for teaching and diminished opportunities for research careers, have resulted in demoralization of faculty, erosion of clinically focused scholarship, and increased concern among medical students, residents, and fellows regarding the feasibility of success in academic and/or research careers. Institutions and professional organizations must consider how to increase the incentives for academic careers so that the next generation of scholars and researchers can be recruited from the best and brightest graduates. The monumental student debt of graduates in psychiatry in the face of diminished reimbursements tends to draw students away from psychiatry toward more procedural and "lifestyle" specialties.

\section{Clinical Learning Environment Factors}

Dramatic changes have occurred over the past two decades in the clinical learning environment in which GME takes place. Traditionally, this environment was largely hospital based and made up of inpatient care teams of residents and fellows who were primarily responsible for the care of "ward patients" (many of whom were indigent or uninsured). These teams were supervised by attending physicians who provided oversight but were not generally involved in direct care (except in operating and delivery rooms and intensive care units). "Private patients" (i.e., patients with insurance) were primarily cared for by private attending physicians during the daytime and were covered by resident teams at night. In this way, clinical training was kept separate from routine care of insured hospital patients. Changes in the rules for hospital reimbursement by Medicare, Medicaid, and private insurers shifted greater responsibility to supervising faculty, reducing the autonomy of residents and fellows and dramatically curtailing the role of medical students in patient care activities. Supervising faculty became obligated to provide direct care to ward patients and to document their activities in patient records for billing purposes. Moreover, the very process by which patient care and treatments were decided shifted away from resident/fellow teams to the "attending of record," whose availability for conducting bedside teaching rounds and supervising resident/fellow activities was greatly reduced.

Although this change may have appeared to benefit patients, who received more direct contact with senior medical staff, in reality, the attending time on inpatient and consult services has been atomized into week-long (rather than month-long) tours of duty. This frequent turnover of attending coverage has led to fragmented patient care and diminished interactions between teachers and learners. It is not uncommon for residents and medical students to report that their interactions with faculty supervisors are brief, truncated, and exclusively task focused. To complicate matters further, the changes in resident work schedules resulting from the imposition of duty hour restrictions, the increase in night float shift, and the introduction of hospitalist physician teams have all but eliminated the closely knit teams that populated the clinical learning environment of the past. This shift, in turn, has greatly diminished opportunities for camaraderie and closeness among faculty supervisors and trainees.

Although inpatient psychiatry units have been protected from some of the trends seen in the general hospital, economic 
pressures on inpatient psychiatry supervisors has led them to spend less time on the wards to fulfill other clinical responsibilities in the hospital or the clinic. In addition, the pressure to shorten hospital stay lengths, increase patient turnover, and reduce ancillary services (e.g., occupational therapy, art therapy) on inpatient psychiatry units has all but eliminated the notion of the therapeutic milieu in which residents and fellows can directly observe and participate in interdisciplinary, teambased care.

Another major change in the clinical learning environment has been the shift to greater time spent at ambulatory care sites. To be sure, ambulatory care has always been an important component of GME, especially in psychiatry, and increasing resident and fellow exposure to a broad array of ambulatory care sites and patient populations is a laudable goal. But as with inpatient care reimbursements, new restrictions on resident autonomy along with greater demands on attending time in outpatient settings have had mixed effects on clinical teaching. On the one hand, greater direct supervision of trainees' interactions with patients has been well received by teachers and learners (and even patients). On the other hand, independent clinical reasoning and decision making for residents and fellows are hampered by these restrictions.

Further changes in the clinical learning environment can be anticipated with the advent of the ACA and its imperatives to serve greater segments of the previously uninsured or underinsured US population. To be sure, the complexities of the ACA, including economic incentives for insurers and providers to integrate care and to reduce costly and ineffective practices, will continue to stimulate innovations in clinical care. The move toward integrated care promises better and more comprehensive approaches to patient health, including mental health. It is hard to predict at this point in time how the costs of education will be factored into future reimbursement rules and how the changes in clinical care delivery will alter GME. It is easy to imagine, for example, that non-physician health providers (e.g., nurse practitioners, psychologists) will be favored over medical trainees and that academic medical centers, hospital systems, and accountable care organizations will try to limit their costs by reducing their numbers of residents and fellows. Moreover, the future of psychiatry departments, psychiatric institutions, and outpatient mental health clinics remains uncertain until the Mental Health Parity and Addiction Equity Act is implemented. One thing is clear, however, economic incentives must be made available to protect the clinical learning environment so as to train sufficient future psychiatrists to meet the nation's growing mental health needs.

The launch of the ACGME Clinical Environment Review (CLER) Program signals a vital new opportunity for clinical educators and health system administrators to define specific institutional goals and objectives to promote resident and fellow education and to establish explicit policies and procedures ("pathways to excellence") in six key areas: patient safety, health care quality, care transitions, supervision, duty hours and fatigue management and mitigation, and professionalism [12]. The CLER Program has been conducting site visits across the USA to solicit feedback from residents and faculty about the extent to which their institutional resources and policies, administrative infrastructure, clinical operations, and related factors are aligned with the objectives of clinical education. In the immediate aftermath of its launch, new opportunities for how to teach about these critical content areas have become a focus of discussion among US medical educators. The hope is that the CLER Program will foster positive changes in the learning environment by giving residents and faculty a greater voice in how institutional resources are allocated on behalf of clinical education.

\section{Social Factors}

As noted earlier, awareness of the prevalence and epidemiology of psychiatric disorders is growing. Beyond the vast array of problems facing youth and families, the aging of the population reveals a renewed urgency for providing greater resources to the much-needed care and treatment of geriatric psychiatric problems. Yet the number of those completing geriatric fellowships is extraordinarily below what is necessary to care for the aging of the population.

The increasing racial, ethnic, and cultural diversity of the population and the numbers of recent immigrants to the USA have raised awareness of the need for improved training in cultural competency. The social acceptance of blended and lesbian, gay, bisexual, and transgender families has added to the imperative for understanding the psychosocial issues of a wider range of families and communities. Many medical students, residents, and fellows are concerned about and interested in global mental health, requiring even more attention to the expression of mental illness and stressors in the broader international context.

Despite the growing awareness of mental health and illness in US society, the stigma of mental illness continues both in the USA and around the world. Efforts such as the Mental Health Parity and Addiction Equity Act of 2008 and the ACA of 2010 are laudable advances, yet profound inequalities in and widespread lack of acceptance of psychiatric disorders stubbornly persist. Psychiatric education continues to be neglected in medical school curricula and in GME training, particularly in primary care medicine and pediatrics - specialties on the frontlines of medical care. The paucity of training in medical school and primary care residencies not only diminishes the general physician's understanding of psychiatric disorders but also devalues psychiatry in the eyes of medical students and residents. Stigma against psychiatric patients and 
against the discipline of psychiatry is continual problems that psychiatric educators must address [13].

\section{Information Technology Factors}

The introduction of information technology into medical education has been slow in comparison with other sectors of society. Whereas the creation of medical databases and online journal access through publishers and the NIH library has provided greater access to medical literature, other technological innovations have been slower to follow. Technologies in research, from computer analysis of large data sets to functional magnetic resonance imaging or the human genome to the creation of neural networks, have all greatly advanced understanding of basic health and disease. This revolution in basic information has challenged not only educators to keep up with scientific discoveries but also practitioners to stay current on developments in the field. Federal legislation (e.g., the Health Insurance Portability and Accountability Act) was required to lay the groundwork for the recording and sharing of personal health information, but financial incentives for "meaningful use" pushed forward the adoption of electronic medical records (EMRs) along with required training of medical students, residents, fellows, and attending physicians on their use. Paper tests have given way to computer exams, slideshows have become electronic presentations, and teleconferencing is used more widely in medical care delivery (especially in psychiatry, which is well suited to telemedicine), but the full potential of information technology in medical education and clinical care has not been reached.

Advances in technology are beginning to have a greater presence in the classroom and the clinic, with simulated patients, apps for medication information, and data entry about patients. Technology has much to offer in transforming educational methods into individualized training for students, residents, fellows, and practitioners. Technology is also being used to organize and measure medical education and patient care. What is now a downpour of data (Milestones, resident and faculty surveys, Press Ganey reports, patient test results, etc.) will become a veritable flood. The central question is what to make of all these metrics and how to use them efficiently and effectively. One new information technology that may help is deep learning, or machine learning, the use of computer algorithms to analyze data and detect patterns and associations that otherwise may not be directly observed. Such techniques have been used in a number of other fields to provide a better understanding of complex systems and are just now being applied in medical settings. Regardless of the role technology will play, its introduction requires additional time devoted to training practitioners in using it appropriately. Technology is also transforming interactions between patients and practitioners and must be factored into medical education.
Interesting new questions about the impact of information technology are being raised. How does the physician avoid having the computer screen distract from the patient? How should physicians handle social media messages and texts from patients? All of these changes will have an impact on medical education and clinical care.

\section{Discussion}

The central unifying theme to the forces shaping current and future medical education is the focus on measured outcomes in both education and patient care. Because separate but overlapping authorities deal with each stage of medical training and continued education, the expectation for documenting and reporting has become burdensome for both the educator and the practitioner. Although some discussion has occurred among different regulatory agencies regarding critical outcomes and sharing of data regarding training programs (e.g., reporting of graduate board pass rates from ABMS to ACGME), further consolidation of standards and information sharing would greatly ease the burden of duplication of reports and multiple assessments across different programs. This integration should not only cross UME, GME, and MOC but also be conducted in an interdisciplinary fashion.

As medical education and clinical care move toward integrated models, there must be assessment tools and techniques that all specialties are familiar with and that can provide meaningful information for any trainee. The ACGME is moving in that direction with the revision of the Milestones stipulating multidisciplinary standards for the shared core competencies of Practice Based Learning and Improvement, Interpersonal and Communication Skills, Systems Based Practice, and Professionalism. It has been very difficult for individual programs to develop the proper assessment tools and to train supervising faculty in their use. In an attempt to deal with the increased demands of assessment data tracking, different private databases have been used, adding to administrative costs. Similar to the different EMRs in use, these databases for medical training vary greatly in their features and make developing common assessment tools difficult. Although, understandably, the regulatory agencies do not engage in a potential conflict of interest in marketing solutions, some way should exist to provide a universal assessment database with appropriate security and oversight to ease the burden on training programs. Such a unified database would enable programs to transmit educational outcomes in a shared, universal platform but would decrease the financial burden of departments in creating their own databases and taking the additional time to put information in formats that regulatory agencies require. The uniformity of such a platform would save costs and administrative burdens on departments. It is important that on a national level, professional organizations overseeing medical 
education support the development, dissemination, and training of evidence-based assessments. This support should include not only publications and presentations at national meetings but also a specific curriculum in faculty development. A common set of assessment tools and databases would greatly facilitate faculty development, promote interdisciplinary training, and enhance further study of training methods.

Technology can provide some relief in the demands for assessment of student and resident competency, allowing for easier and real-time evaluation and data entry. More research is needed into what measures provide the best information and how to interpret data on trainee performance. The Milestones were based on professional consensus but may not reflect the actual developmental progression of physician skills. Discovering which skills are essential may provide simpler assessments, but research in this aspect of training is lacking. The application of new analytic techniques may provide answers to the question of what data are most important in assessing student, resident, and fellow performance. Attention must also be paid to the application of information technology in patient care and training of students and residents in the proper use of the EMR, online communication, and social media. Some of the regulatory agencies have supported research along these lines, but more must be done to fund these studies, disseminate their findings, and recommend evidence-based policies to guide future reforms. UME and GME need reliable and valid tools to evaluate competencies.

Closer attention must also be paid to the salient features of the clinical learning environment, especially to those factors influencing teacher-learner interactions and clinical care team dynamics. In preparing trainees to work in more integrated care environments with colleagues from other specialties and professions, psychiatric educators must model quality collaborative care in their institutions. Clearly, both the process and the content of medical education are determined by the conditions under which patient care is provided and by the degree to which institutions respect and value their practitioners. Concern about "burnout" among residents, fellows, and attending physicians is growing in academic medical centers. Psychiatric educators have an important role to promote wellness and resilience for learners at all levels of training and for faculty members as well.

Institutional leadership must recognize the increased workload for administration of quality medical training and provide protected time not only for assessing and reporting trainee progress but also for developing faculty in new training methods. The growing and necessary oversight of medical education has resulted in unfunded mandates for all training programs. Institutions and departments require new funding mechanisms for protected teaching time and for faculty development. National, regional, and local means for such funding must be considered in support of these changes. There have been calls for the federal government to get out of subsidizing medical education, but as long as the public expects quality care and the role of the federal government in providing health care, public support for training is necessary. The financial and system pressures for clinical productivity are great, but the need for quality medical education is essential to meet the challenges of an expanding population.

\section{Compliance with Ethical Standards}

Disclosures On behalf of all the authors, the corresponding author states that there are no conflicts of interest.

\section{References}

1. Joyner BD. An historical review of graduate medical education and a protocol of Accreditation Council for Graduate Medical Education compliance. J Urol. 2004;172:34-9.

2. Hollender M. The founding of the ABPN. In: Aminoff MJ, Faulkner LR, editors. The American Board of Psychiatry and Neurology: looking back and moving ahead. 1st ed. Arlington: American Psychiatric Publishing; 2012. p. 9-16.

3. Nasca TJ. The CEO's first column: the next step in the outcomesbased accreditation project. ACGME Bull. 2008;5:2.

4. Hawkins RE, Lipner RS, Ham HP, Wagner R, Holmboe ES. American Board of Medical Specialties maintenance of certification: theory and evidence regarding the current framework. J Contin Educ Health Prof. 2013;33 Suppl 1:S7-19.

5. Ulmer C, Wolman D, Johns M (Eds.). Resident duty hours: enhancing sleep, supervision, and safety. Committee on Optimizing Graduate Medical Trainee (Resident) Hours and Work Schedules to Improve Patient Safety for the Institute of Medicine. Washington, DC: National Academies Press; 2008

6. Miller P, Mosley K. Physician reimbursement: from fee-for-service to MACRA, MIPS and APMs. J Med Pract Manag. 2016;31:266-9.

7. Nasca TJ, Philibert I, Brigham T, Flynn TC. The next GME accreditation system — rationale and benefits. N Engl J Med. 2012;366:1051-6.

8. Dall T, West T, Chakrabarti R, Iacobucci W (IHS Inc.). The complexities of physician supply and demand: projections from 2014 to 2025. 2016 update. Prepared for Association of American Medical Colleges. Washington, DC: Association of American Medical Colleges; 2016

9. Ludmerer KM. Let me heal: the opportunity to preserve excellence in American medicine. New York: Oxford University Press; 2015. p. xii.

10. Bagalman E, Napili A. Prevalence of mental illness in the United States: data sources and estimates. Report prepared for Members and Committees of Congress. Washington, DC: Congressional Research Service; 2015.

11. H.R.2646 Helping Families in Mental Health Crisis Act of 2016. Available at https://www.congress.gov/bill/114th-congress/housebill/2646. Accessed 16 Sep 2016

12. Accreditation Council for Graduate Medical Education Clinical Learning Environment Review Evaluation Committee. CLER pathways to excellence: expectations for an optimal clinical learning environment to achieve safe and high quality patient care. Accreditation Council for Graduate Medical Education. 2014. Available at http://www.acgme.org/Portals/0/PDFs/CLER/CLER Brochure.pdf Accessed 16 September 2016

13. Schlozman S, Beresin EV, Balon R, Coverdale JH, Brenner AM, Louie AK, et al. Stigma and mental health: a proposal for next steps. Acad Psychiatry. 2016;40:735-9. 